\title{
Soft $x$-ray detection for small satellites with a commercial CMOS sensor at room temperature
}

\author{
Steve Tammes ${ }^{\mathrm{a}, *}$, Tyler Roth ${ }^{\mathrm{a},{ }^{*}}$, Philip Kaaret ${ }^{\mathrm{a}}$, Casey DeRoo ${ }^{\mathrm{a}}$, Abdallah Elmaleh ${ }^{\mathrm{a}}$, Jessica L. \\ McChesney $^{\mathrm{b}}$, Fanny Rodolakis ${ }^{\mathrm{b}}$ \\ ${ }^{a}$ Department of Physics and Astronomy, University of Iowa, Iowa City, IA 52242, USA \\ ${ }^{\mathrm{b}}$ Argonne National Laboratory, 9700 South Cass Avenue, Argonne, Illinois 60439, USA
}

\begin{abstract}
Recently CMOS (complementary metal-oxide-semiconductor) sensors have progressed to a point where they may offer improved performance in imaging x-ray detection compared to the CCDs often used in x-ray satellites. We demonstrate $\mathrm{x}$-ray detection in the soft x-ray band $(250-1700 \mathrm{eV})$ by a commercially available back-illuminated Sony IMX290LLR CMOS sensor using the Advanced Photon Source at the Argonne National Laboratory. While operating the device at room temperature, we measure energy resolutions (FWHM) of $48 \mathrm{eV}$ at $250 \mathrm{eV}$ and at 83 $\mathrm{eV} 1700 \mathrm{eV}$ which are comparable to the performance of the Chandra ACIS and the Suzaku XIS. Furthermore, we demonstrate that the IMX290LLR can withstand radiation up to $17.1 \mathrm{krad}$, making it suitable for use on spacecraft in low earth orbit.
\end{abstract}

Keywords: CMOS Image sensor, Back-side illuminated, x-rays spectroscopy, x-ray Imaging, Silicon, Radiation Effects, Sensor performance.

*Steve Tammes, steven-tammes@uiowa.edu

*Tyler Roth, tyler-roth@uiowa.edu

\section{Introduction}

The availability of low-cost small satellites for astrophysics drives a need for low-cost detectors that place minimal demands on the limited spacecraft resources available. ${ }^{1}$ The charge-coupled device (CCD) detectors often used for x-ray detection on space-based x-ray observatories have high cost and require cooling to temperatures near $-100 \mathrm{~K} .^{2-4}$ Complementary Metal-Oxide-Semiconductor (CMOS) sensors are designed for operation at room temperature and many commercial devices are available at low cost. The active pixel design of CMOS sensors enables lower power consumption, faster readout rates, and comparable noise levels to CCDs. ${ }^{5}$

Here, we investigate the candidacy of a low-cost commercial CMOS sensor as an $\mathrm{x}$-ray spectrometer in low earth orbit (LEO) for future small satellite missions. In Sec. 2, we describe the selected CMOS sensor, initial results on $\mathrm{x}$-ray detection, and our $\mathrm{x}$-ray event processing algorithm. 
We characterize the soft x-ray performance of the sensor using measurements obtained at an x-ray synchrotron beamline at the Argonne National Laboratory in Sec. 3. We then discuss the electron noise (Sec. 4), radiation testing (Sec. 5), and our conclusions (Sec. 6).

\section{2 x-ray Detection And Event Processing}

\section{1 - -ray Detection}

Back-illuminated (BI) sensors can provide superior performance for soft x-ray detection because the photons do not pass through the passivation, metallization, and inter-dielectric layers that form the pixel electronics. We evaluated several candidate BI sensors and selected the Sony IMX290LLR CMOS sensor for additional study due to its low electronic noise which is important in achieving good spectral resolution for soft x-rays. The IMX290LLR contains a 1936×1096 array of $2.9 \mu \mathrm{m}$ square back-side-illuminated pixels and can achieve frame rates of up to $135 \mathrm{fps}$. To minimize development costs, we used an IDS Imaging Development Systems (https://en.idsimaging.com/) UI-3860-LE-M-GL camera, that includes an IMX290 sensor and circuitry to provide a USB interface, and the associated Software Development Kit (SDK). For x-ray detection, we removed the coverglass designed to protect the sensor's surface.

Our initial x-ray tests were performed using an ${ }^{55} \mathrm{Fe}$ radioactive source and an $\mathrm{x}$-ray tube fluorescing a teflon target and the walls of the vacuum chamber where the sensor was mounted. This produces characteristic emission lines from several elements including $\mathrm{F}, \mathrm{Cr}, \mathrm{Mn}, \mathrm{Fe}$, and bremsstraulung continuum radiation. The chamber was evacuated to $\approx 10^{-2}$ Torr and the sensor was mounted on a chiller plate maintained at a constant temperature of $21^{\circ} \mathrm{C}$. We acquired 4,000 images each with $300 \mathrm{~ms}$ exposure under x-ray illumination and 100 frames with no x-rays present 


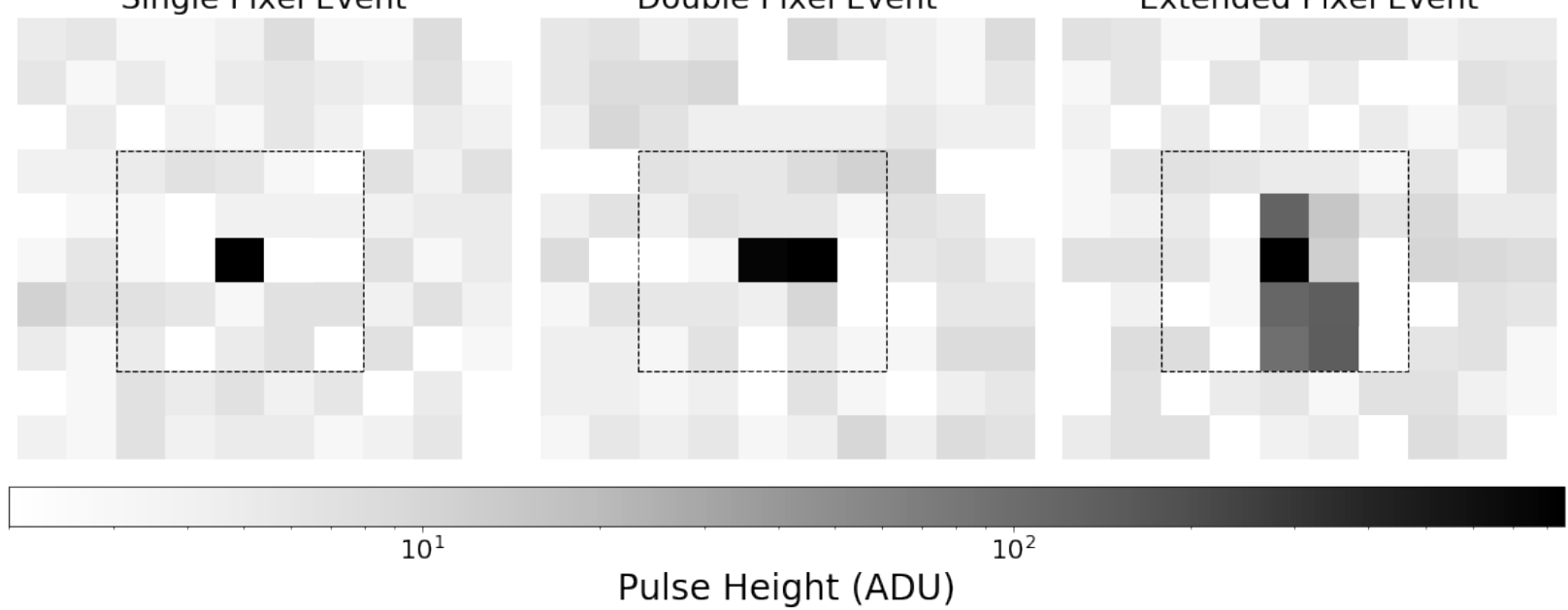

Fig 1: Cutout images of the three types of events we consider for event processing. Shown from left to right are single pixel, double pixel, and extended pixel events. These events correspond to energies of $3.3 \mathrm{keV}, 3.1 \mathrm{keV}$, and $5.4 \mathrm{keV}$ respectively.

for dark and bias measurements. Cutouts of $\mathrm{x}$-ray events taken from single frames are shown in Fig 1. x-rays produce events in which charge is deposited in one, two, or multiple pixels.

To select an appropriate gain for the sensor, we collected a series of spectra using the ${ }^{55} \mathrm{Fe}$ radioactive source at different gain settings. We measured the centroid of the pulse height distribution for the Mn K- $\alpha$ emission line (corresponding to $1620 e^{-}$for an average hole-pair creation energy of $w=3.64 \mathrm{eV}$ in silicon at room temperature ${ }^{6}$ ) using the event processing methodology outlined in Sec 2.2, and adopted settings producing a gain and associated fitting error of $0.991 \pm$ $0.006 \mathrm{ADU} / \mathrm{e}^{-}$. These gain settings were used for all of the data described below.

\subsection{Event Processing}

Individual x-rays may deposit charge in multiple pixels due to the finite extent of the track traversed by primary photoelectron, the ejection of Auger electrons, and charge diffusion. To cope with the variable spread of charge across pixels, the event processing algorithms used for x-ray CCDs, such as Chandra's Advanced CCD imaging spectrometer (ACIS), search for pixels with charge greater 
than the 'event threshold' and then record values in the surrounding $3 \times 3$ pixel grid. Pixels above the 'split pixel threshold' and sharing a side with the central pixel or in a $2 \times 2$ square including the central pixel are included in the sum used to reconstruct the energy of the incident photon. ${ }^{3}$ As shown in Fig. 1, the X-ray events from the IMX290 sometimes extend over a larger range of pixels. This is likely due to the relatively small size of the pixels. We have developed an event processing algorithm utilizing a $5 \times 5$ grid of pixels.

Before processing the images, we first identified anomalous (hot) pixels. We started by collecting 100 dark frames of the same exposure time as the data. Setting a threshold equal to 2 standard deviations above the median value of the 100 dark frames, we marked all pixels that are above the threshold in $10 \%$ or more of the frames as hot. These pixels were excluded in further processing. For the data described below, $0.47 \%$ of the pixels were marked as hot. A master dark frame was compiled by taking the mean of the dark frames. The master dark frame was subtracted from each of the x-ray frames.

The dark-subtracted x-ray frame is then scanned. For each pixel above the event threshold, a surrounding $5 \times 5$ grid is defined. The set of pixels above the split pixel threshold ('hit pixels') that are connected to the central pixel by a chain of adjoining hit pixels are included in the sum used to estimate the photon energy and the position of the central pixel is recorded as the x-ray position. The event and split pixel thresholds were optimized by examining pulse height spectra for x-rays with energies between $250 \mathrm{eV}$ and $1700 \mathrm{eV}$ (see Sec. 3). The thresholds were adjusted to minimize the width and skew of the pulse height distributions. We found an optimal event threshold of 26 ADU and split pixel threshold of 5 ADU. 


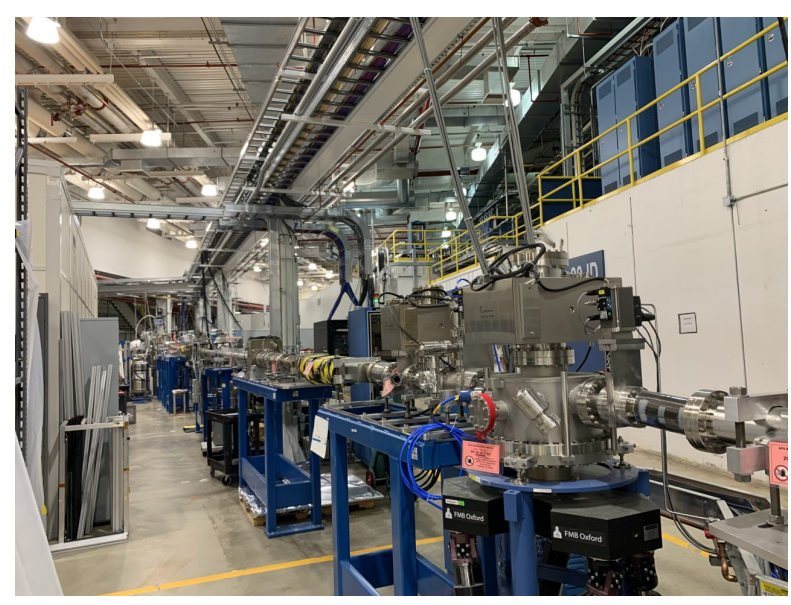

(a)

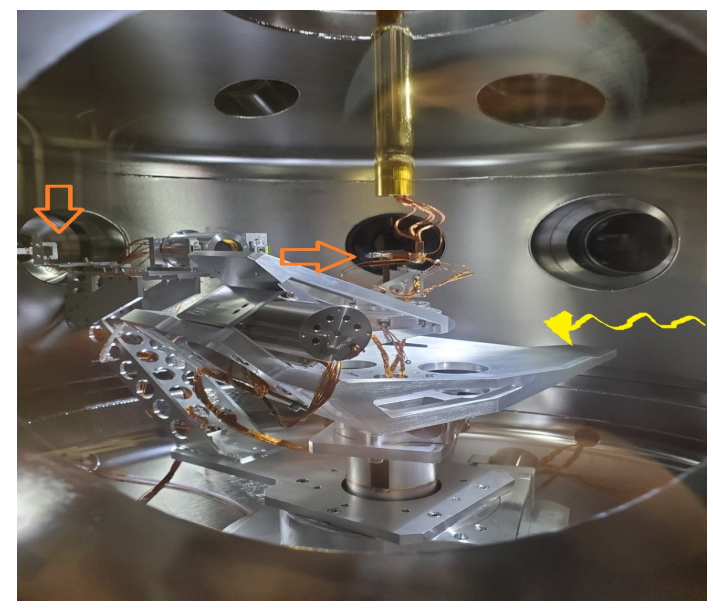

(b)

Fig 2: (Left) The beamline used at APS for the experiment. (Right) Vacuum chamber where the sensor was held. Arrows from left to right show the sensor port, Si wafer, and beam direction.

\section{Argonne Data}

\subsection{Experimental Setup}

In December 2019, we tested the sensor at the Intermediate Energy X-ray (IEX) beamline 29-ID of the Advanced Photon Source (APS) at the Argonne National Laboratory in Illinois. This beamline utilizes an electromagnetic, variably polarizing undulator source followed by a monochromator and is capable of producing monochromatic x-rays between $250-2500 \mathrm{eV} .^{7}$ Images of the experimental setup are shown in Fig 2a and Fig 2b. Our sensor is not capable of handling the intensity of the direct beam in photon counting mode, so it was mounted on a port at an angle $35^{\circ}$ with respect to the incident beam. A pump near the sensor maintained a high vacuum $\left(\approx 10^{-9}\right.$ Torr $)$. A Si wafer was used to reflect the incident beam thereby reducing the beam flux $\left(\sim 10^{12} \gamma / \mathrm{s}\right)$ to a level that could be handled by the camera $(<100 \gamma / \mathrm{s})$. Some experimentation was required to focus the beam in the center of the sensor and the optimal graze angle was $17.5^{\circ}$.

With the beam centered on the sensor and the vacuum chamber covered to minimize ambient light on the sensor, we accumulated sensor images with an exposure time of $300 \mathrm{~ms}$ at energies 


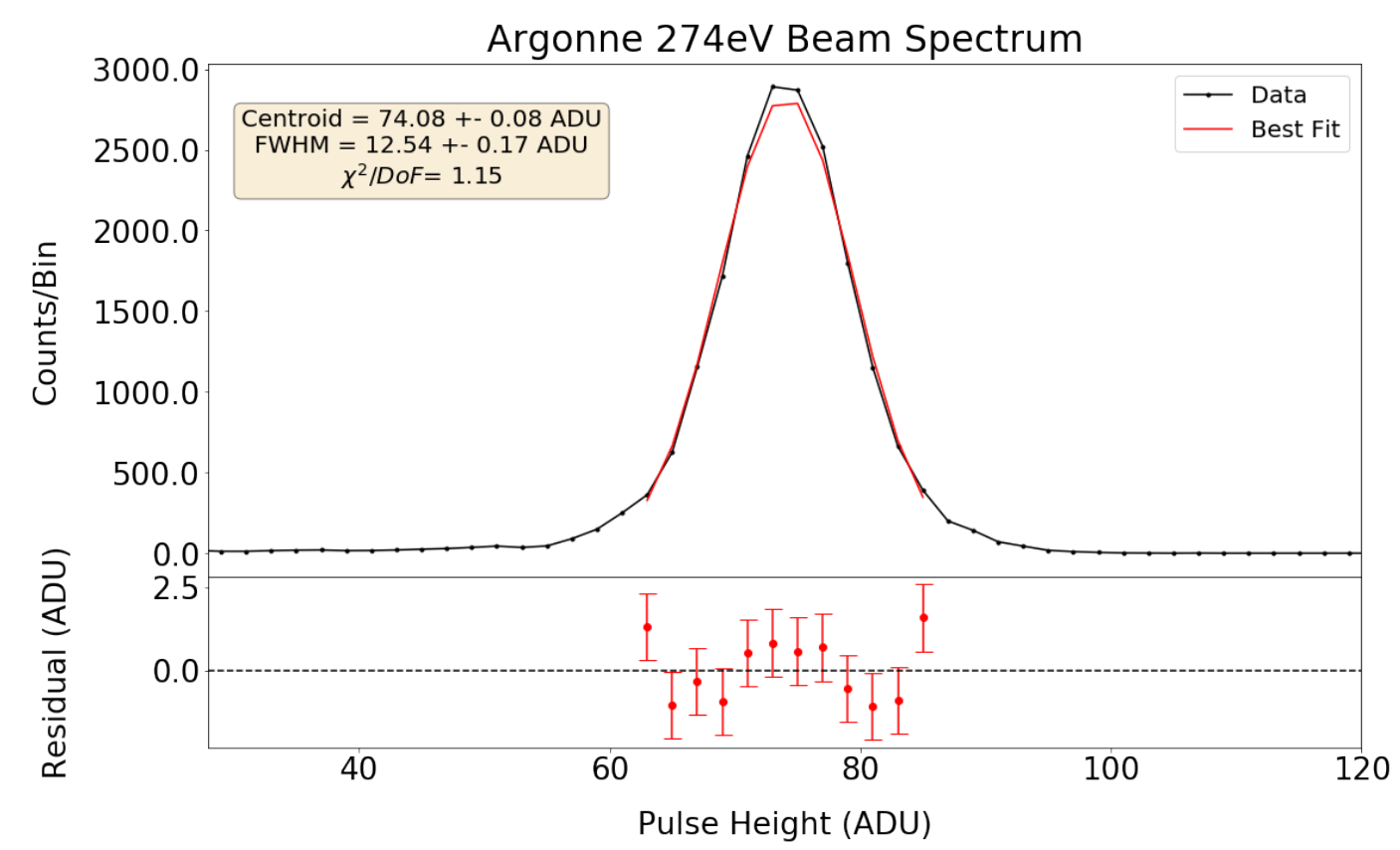

Fig 3: Example of a fitted spectrum from a beamline energy of $274 \mathrm{eV}$ with the best fit Gaussian model.

from $250 \mathrm{eV}$ to $1700 \mathrm{eV}$ (see Table 1). At each energy, we accumulated frames until at least 20,000 photon events were registered. The sensor maintained an average temperature of $25^{\circ} \mathrm{C}$ during this continuous run. Both before and after taking beamline data, we turned off the beam and acquired 100 dark frames with $300 \mathrm{~ms}$ exposure.

\subsection{Spectral Fitting}

Using the algorithm described in Sec. 2.2, x-ray events were extracted from the sensor images. The list of x-ray events was then used to produce an energy spectrum at each beam energy using a bin width of 2 ADU/bin. We fitted each spectrum with a Gaussian model over a pulse height band covering the peak of the Gaussian, as seen in the solid line in Fig 3.

The error on the counts in each pulse height bin was calculated as the quadrature sum of the Gehrels variance function ${ }^{8}$ and a linear term due to differential non-linearity (DNL) proportional to the counts in each bin. The same DNL factor was used for all spectra and was adjusted until the 


\begin{tabular}{ccccc}
\hline $\begin{array}{c}\text { Energy } \\
(\mathrm{eV})\end{array}$ & $\begin{array}{c}\text { Centroid } \\
(\text { ADU })\end{array}$ & $\begin{array}{c}\text { FWHM } \\
(\text { ADU })\end{array}$ & DoF & $\chi_{\nu}^{2}$ \\
\hline 250.0 & $67.0 \pm 0.1$ & $12.4 \pm 0.2$ & 8 & 1.7 \\
274.0 & $74.1 \pm 0.1$ & $12.5 \pm 0.2$ & 9 & 1.2 \\
280.0 & $74.9 \pm 0.1$ & $12.6 \pm 0.2$ & 8 & 0.7 \\
400.0 & $108.4 \pm 0.1$ & $14.7 \pm 0.1$ & 10 & 0.5 \\
574.0 & $155.0 \pm 0.1$ & $15.2 \pm 0.2$ & 11 & 1.2 \\
950.0 & $257.0 \pm 0.1$ & $16.7 \pm 0.1$ & 12 & 0.5 \\
1200.0 & $325.2 \pm 0.1$ & $18.2 \pm 0.1$ & 13 & 0.5 \\
1450.0 & $392.6 \pm 0.1$ & $20.0 \pm 0.2$ & 15 & 0.7 \\
1700.0 & $459.7 \pm 0.2$ & $21.9 \pm 0.4$ & 18 & 2.5 \\
\hline
\end{tabular}

Table 1: Summary of fit values obtained with a Gaussian plus a constant model.

fits produced $\chi_{\nu}^{2} \approx 1$ giving a value of 0.045 . We interpret this as the DNL of the analog-to-digital converters in the IMX290. Table 1 shows the best fit parameters for each spectrum.

\subsection{Energy Calibration}

We performed a linear regression of the best fitted Gaussian centroids versus beam energy as shown in Fig. 4. We inverted the relation to find the pulse height to energy calibration. We obtained a best fit slope of $3.6877 \pm 0.0008 \mathrm{eV} / \mathrm{ADU}$ and intercept of $1.840 \pm 0.005 \mathrm{eV}$ yielding the following conversion function:

$$
\text { Energy }(\mathrm{eV})=\frac{3.69 \mathrm{eV}}{\mathrm{ADU}}(\mathrm{ADU} \text { value }+1.8 \mathrm{eV})
$$

The slope is equivalent to a camera gain of $0.9870 \pm 0.0002 \mathrm{ADU} / e^{-}$. This is more accurately determined than our initial gain calibration done with the radioactive ${ }^{55} \mathrm{Fe}$ source and consistent within the uncertainties of that measurement.

With active pixel technology, some variations of gain across the regions of the sensor can be expected. To quantify the variations in gain across the sensor, we divided the sensor area into a $5 \times 5$ 


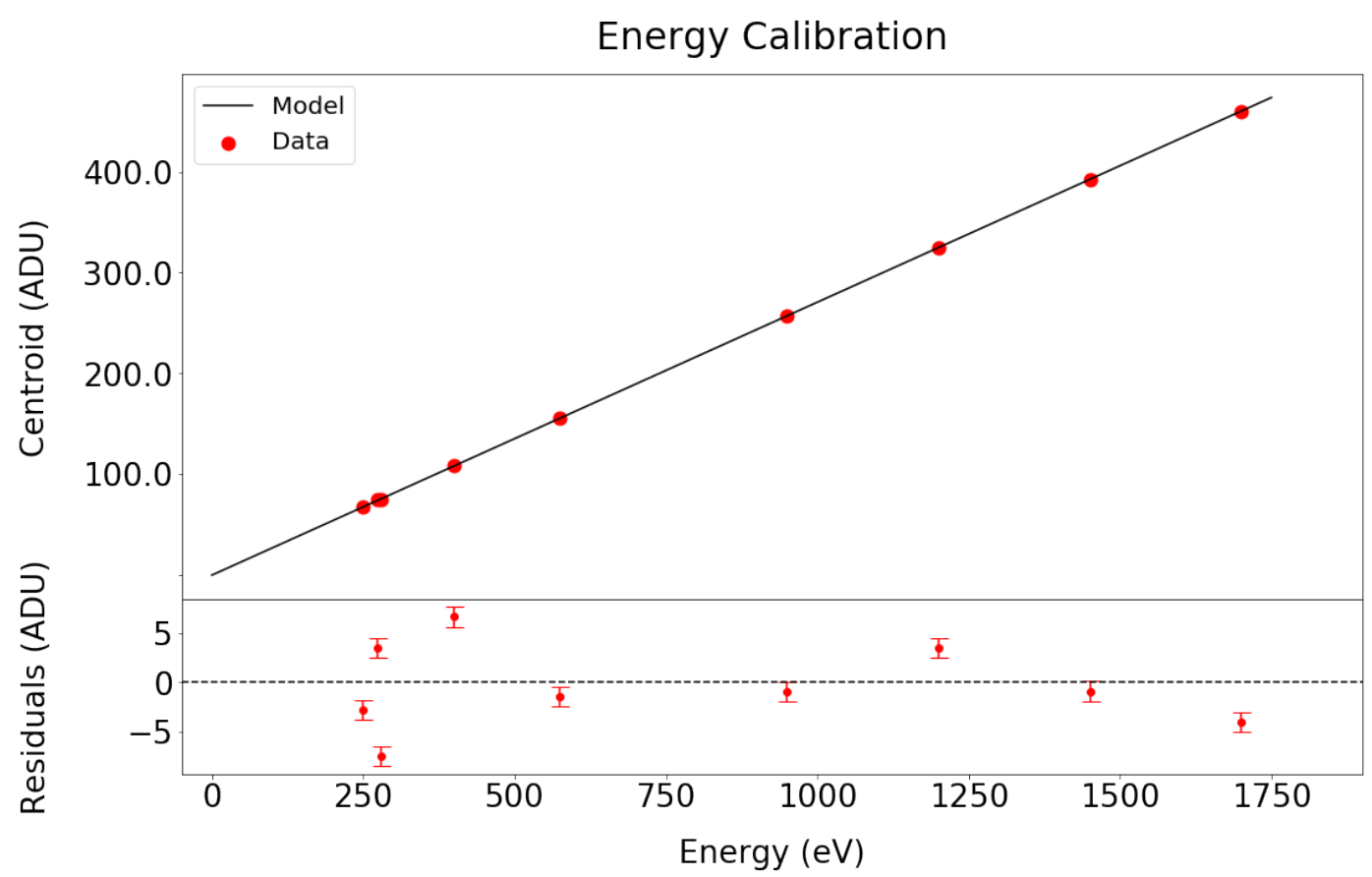

Fig 4: Best fit linear regression to the centroid of the Argonne IEX APS lines with residuals plotted in the lower figure.

grid, each grid 387 by 219 pixels. We then calculated the gain within each grid by fitting only the events that occurred within the grid and performing the same linear regression described above. We determined an average gain weighted by the slope error in each grid region, which we find to be $3.686 \pm 0.005 \mathrm{ADU} / \mathrm{eV}$. The gain varies by $0.14 \%$ across the sensor and we choose to use a single gain for the whole sensor.

In Fig. 5 we display calibrated spectra from various beam energies on the same plot. The Gaussians were normalized to have the same area under their curves.

\subsection{Energy Resolution}

The IEX resolving power is $10^{4}$; thus, the finite widths of the spectral peaks are due to the sensor. Fig. 6 shows the energy resolution in terms of the Gaussian full width half max (FWHM) as a function of beam energy. We modeled the energy resolution as the quadrature sum of the Fano limited Poisson fluctuations in the number of photoelectrons produced per photon and the electronic noise 


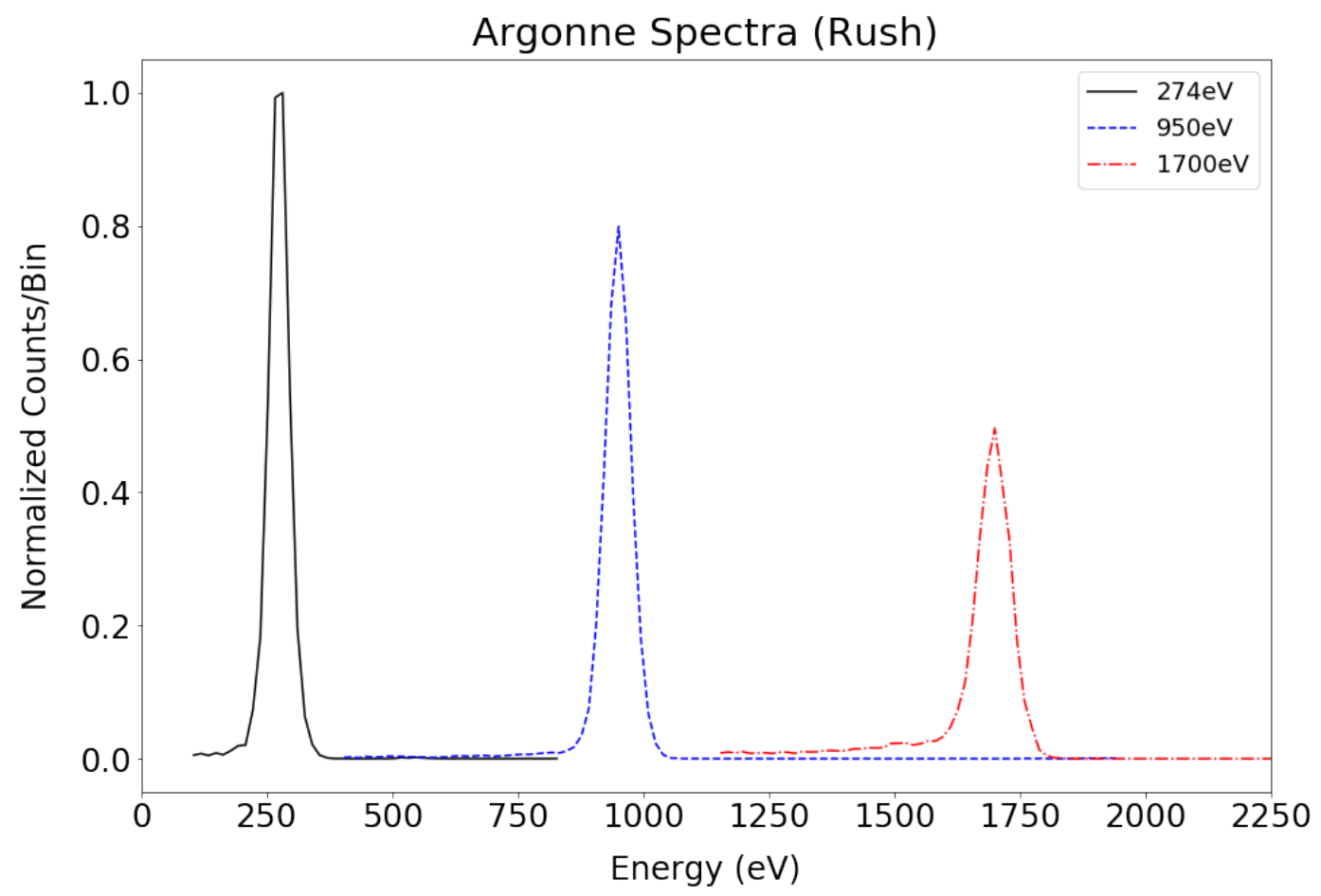

Fig 5: Energy calibrated spectra with a y-axis normalization at beamline energies of $274 \mathrm{eV}$, $950 \mathrm{eV}$, and $1700 \mathrm{eV}$. There are no significant counts below $400 \mathrm{eV}$ and $1150 \mathrm{eV}$ for the $950 \mathrm{eV}$ and $1700 \mathrm{eV}$ lines respectively. We do not plot these spectra below these thresholds for visual clarity.

per pixel,

$$
\mathrm{FWHM}=2.355 w \sqrt{N \sigma^{2}+\frac{f E}{w}}
$$

where $w=3.64 \mathrm{eV}$ is the average ionization energy of silicon, $f=0.115$ is the Fano factor for silicon, ${ }^{9} N$ is the number of pixels used to calculated the pulse height sum for each event, and $\sigma$ is the root-mean-square (RMS) electronic noise per pixel.

Fig. 7 shows the average number of pixels used in the pulse height summation as a function of energy. The error bars indicate the standard deviation. We attempted two different variations of $N$ for fitting, the first uses a constant median $N$ value of 2 and the second uses the variable mean number of pixels as a function of energy as shown in Fig. 5. We allowed the electronic noise $\sigma$ 
Energy Resolution

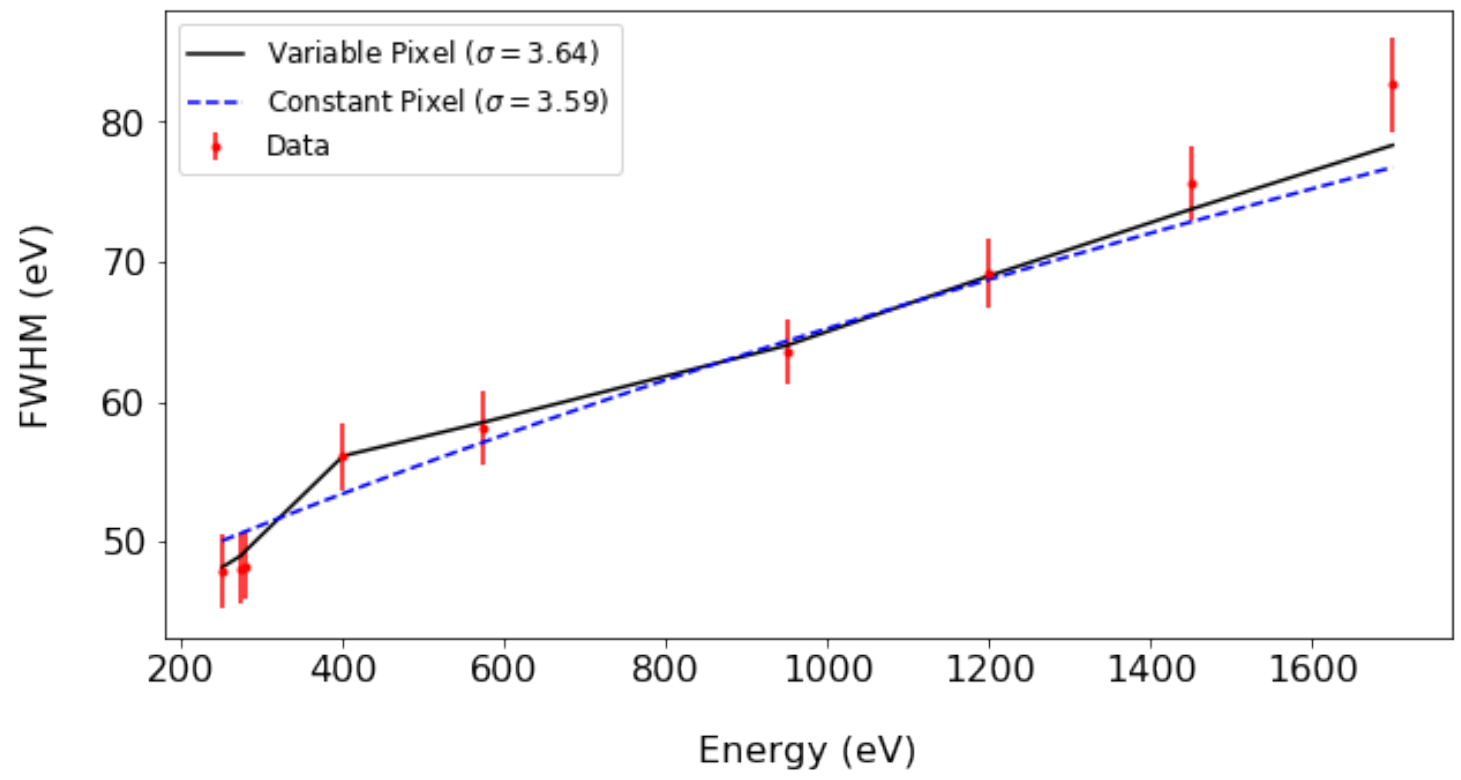

Fig 6: Energy resolution (FWHM) modeled by Eq 2.

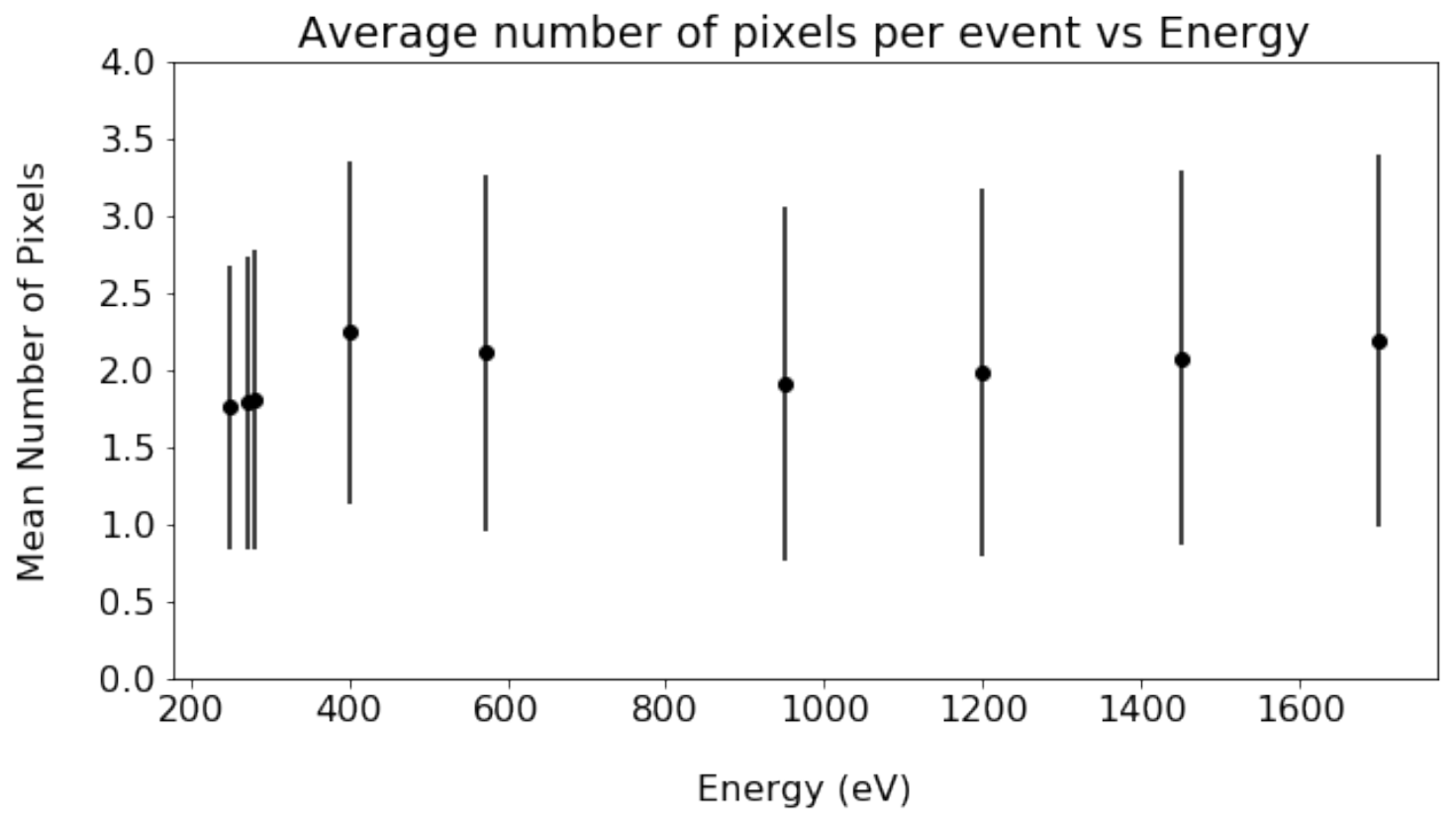

Fig 7: Mean number of summed pixels versus energy.

parameter to vary and fit the two models to the resolution curve in Fig. 7. We found best fit values of $3.64 \pm 0.05 e^{-}$and $3.59 \pm 0.10 e^{-}$for the variable and constant pixel models, respectively. Our FWHM resolution values of $48.1 \mathrm{eV}$ at $274 \mathrm{eV}$ and $75.6 \mathrm{eV}$ at $1450 \mathrm{eV}$ are competitive with the values of $\sim 50 \mathrm{eV}$ and $\sim 70 \mathrm{eV}$ near $0.3 \mathrm{keV}$ and $1.5 \mathrm{keV}$, respectively, for the back-side illuminated 


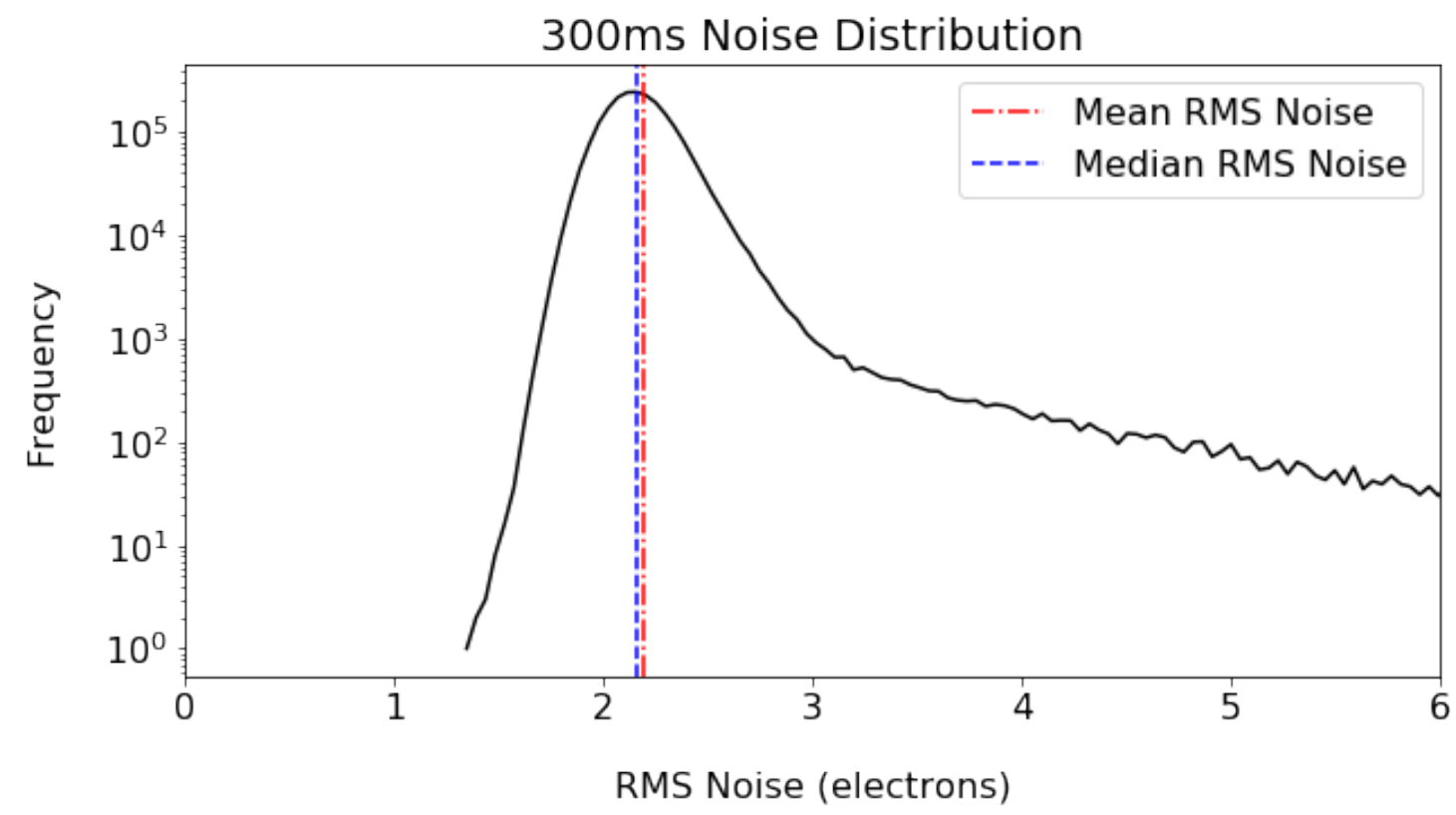

Fig 8: RMS noise distribution of the IMX290LLR chip at $300 \mathrm{~ms}$. The vertically and nonvertically dashed lines correspond to the mean and median of the distribution respectively.

CCDs aboard the Suzaku x-ray Imaging Spectrometer, ${ }^{4,10}$ and also with the resolution of $96 \mathrm{eV}$ at $1.49 \mathrm{keV}$ reported for the ACIS CCDs. ${ }^{11}$

We fitted the Mn K- $\alpha$ and Mn K- $\beta$ lines in the spectrum mentioned in Sec 2. The energy resolution values are larger than those estimated using Eq 2 and the best fit noise for energies below $2 \mathrm{keV}$. The degradation of the energy resolution above $2 \mathrm{keV}$ may be due to incomplete charge collection. Significant degradation of energy resolution at high energies has been found in a front-illuminated sensor and attributed to a reduction in charge collection efficiency for X-ray that penetrate into the $p^{+}$substrate beneath the epitaxial layer. ${ }^{12}$ This preferentially occurs for higher energy X-rays since they have longer absorption depth. The $p^{+}$layer is absent in back-illuminated devices, but incomplete charge collection may occur in the pixel circuitry layer or the carrier wafer. 


\section{Noise Measurements}

The sensor noise can be measured by examining the average variance in each pixel when a sensor is subject to no light at short exposures. Due to the construction of the CMOS pixel, namely that each pixel contains its own circuitry, the RMS noise varies between pixels. This is different than a CCD detector which traditionally has a single amplifier and analog-to-digital converter for all pixels and therefore maintains uniform noise for all pixels.

We evaluated the noise of the IMX290 by taking a series of 100 frames with the sensor in the dark in a vacuum chamber. Each image had an exposure $300 \mathrm{~ms}$ and the sensor was held at a constant temperature of $21^{\circ} \mathrm{C}$. We constructed a master dark frame by taking the mean ADU value in each pixel of the 100 images and then subsequently subtracting the master image from each individual one.

We constructed a distribution of the per pixel noise by taking the RMS of the 100 master subtracted images (Fig. 8). We find mean and median read noise values of $2.18 e^{-}$and $2.17 e^{-}$, respectively. The tail extending to large read noise of the distribution is well known for CMOS sensors and has been characterized as Random Telegraph Signal (RTS) noise. RTS is generated from defects within the silicon that lead to increased electron entrapment and signal variance upon readout. ${ }^{13}$ Fewer than $0.2 \%$ of the pixels have an RMS noise greater than $4 e^{-}$.

\section{Radiation Testing}

We irradiated a IMX290LLR sensor with gamma rays to study its performance in a radiation environment. The testing was done at the RadCore facility at the University of Iowa, see Fig.9. The camera was exposed to gamma-rays from Cs-137 (main peak at $662 \mathrm{keV}$ ) at a rate of 0.054 $\operatorname{krad}(\mathrm{Si}) /$ minute. We operated the camera, recording $10 \mathrm{~ms}$ frames at the same gain settings as 


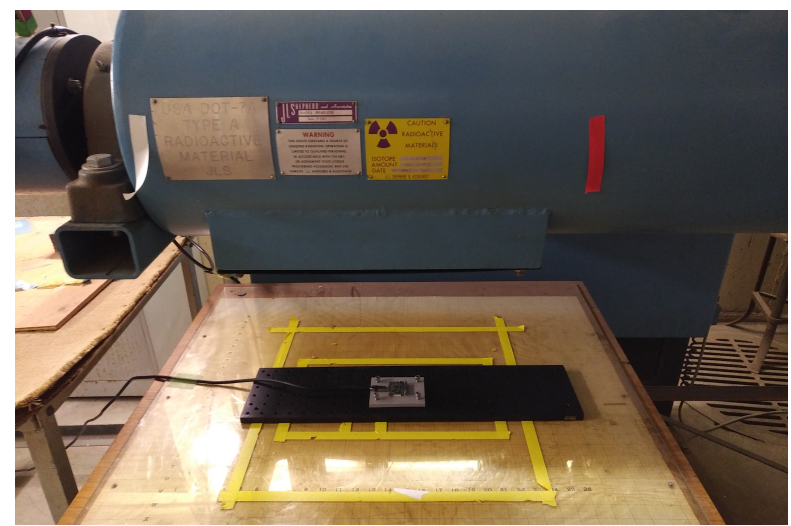

(a)

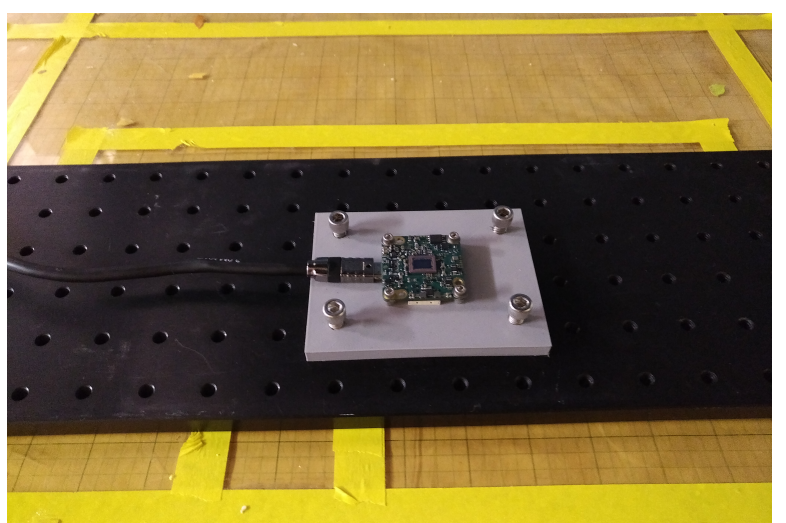

(b)

Fig 9: The IMX290LLR sensor at RadCORE. (Left) The sensor underneath the Cs-137 sample. (Right) Close up of mount securing sensor

discussed above, while monitoring the current drawn by the camera via its USB connection from a radiation shielded location. While the camera was operating correctly, the current was $0.16 \mathrm{~A}$. We ended the test after the current dropped to $0.02 \mathrm{~A}$ and the camera simultaneously ceased to function. The camera functioned correctly while exposed to radiation for a total of 314.8 minutes, giving a total exposure of $17.1 \mathrm{krad}$ before failure. This is a lower bound on the radiation tolerance of the sensor since some other component on the camera may have failed.

Fig. 10 shows the median ADU value versus accumulated dose. The ADU value at the start of the test corresponds to charge deposition of $\sim 20,000 e^{-} / \mathrm{s}$. The median ADU drops by $\approx 15 \%$ during accumulation of the first $6 \mathrm{krad}$, recovers by $\approx 4 \%$ during a 13 minute interval during which the irradiation was stopped, and then shows a more gradual decrease during the remaining testing. The variations may be due to ionization damage of the gate oxide in the active pixel circuity. ${ }^{14}$ Such damage anneals at room temperature, consistent with the recovery while irradiation was stopped. Ionization damage depends on rate of irradiation which was much higher during the test than expected in a typical low Earth orbit (LEO), e.g. $\sim 5$ krad over 2 years. Thus, testing at a low dose rate would be of interest. We note that the sensor continued to operate as the median ADU 


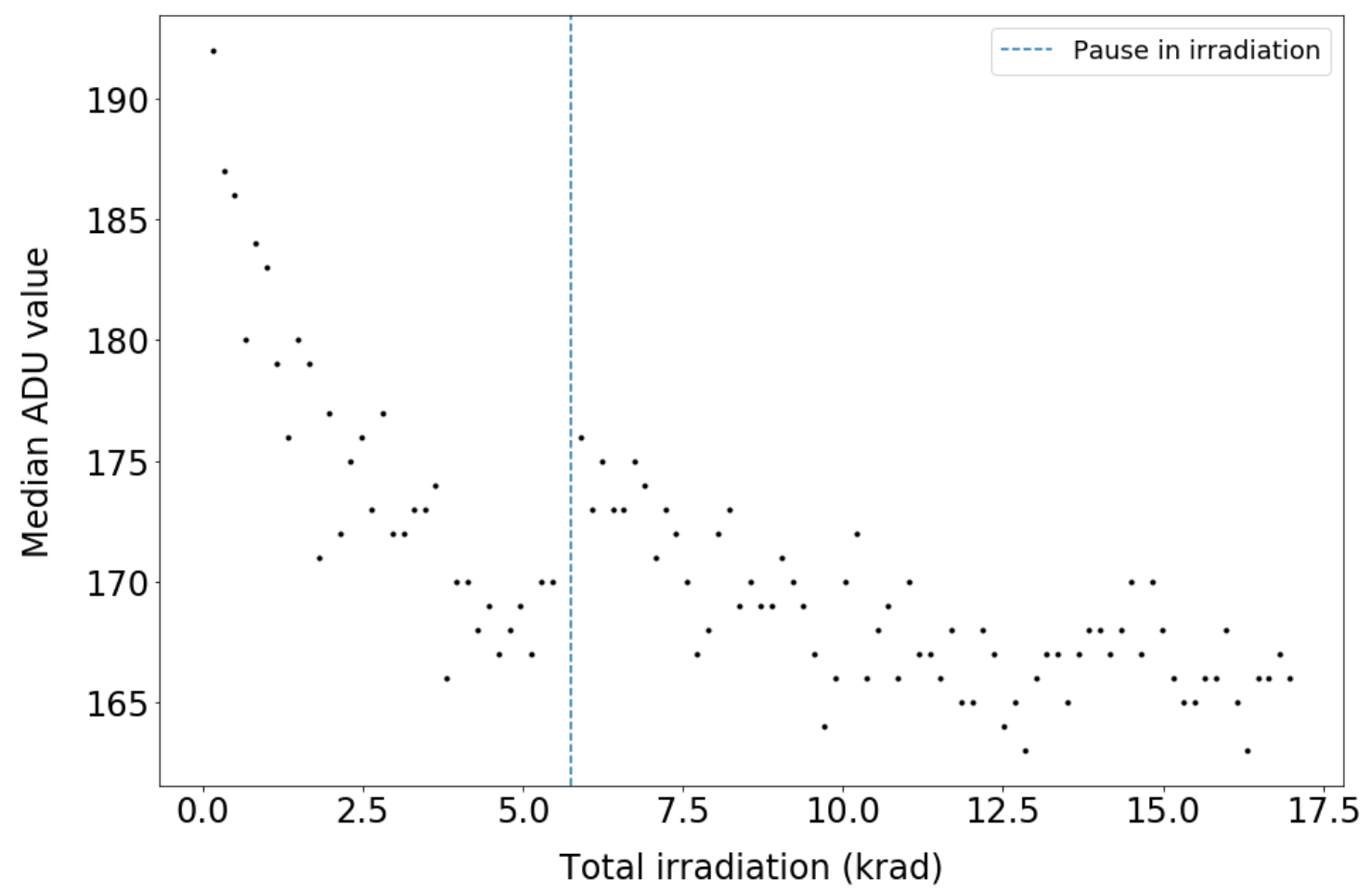

Fig 10: Median ADU value versus accumulated dose

decreased. Thus, the sensor would likely continue to function, although perhaps with some change in the energy calibration, over a moderate duration mission of several years in LEO.

\section{Conclusion}

We have characterized the performance of a commercial Sony IMX290LLR CMOS sensor as an imaging x-ray spectrometer intended for small satellites. Radiation testing shows that the sensor and accompanying electronics is operational for well beyond the radiation doses expected for missions of two years duration in low Earth orbit. Testing at a synchrotron x-ray beamline demonstrates the back-illuminated CMOS sensor operated at temperatures near $+20^{\circ} \mathrm{C}$ has an energy resolution competitive with the cooled CCD sensors on current $\mathrm{x}$-ray observatories, enabling use of such sensors in the resource-limited environment of small spacecraft. 
Acknowledgments

We thank Amanda Kalen of RadCore for her assistance with the radiation testing. This research was supported in part by the Iowa Space Grant Consortium under NASA Award No. NNX16AL88H. This research used resources of the Advanced Photon Source, a U.S. Department of Energy (DOE)

Office of Science User Facility operated for the DOE Office of Science by Argonne National Laboratory under Contract No. DE-AC02-06CH11357; additional support by National Science Foundation under Grant No. DMR-0703406.

\section{References}

1 P. Kaaret, K. Jahoda, and B. Dingwall, "HaloSat-A CubeSat to Study the Hot Galactic Halo," HEAD , 116-21 (2014).

2 M. J. Turner, A. Abbey, M. Arnaud, et al., "The European photon imaging camera on XMMNewton: the MOS cameras," Astronomy \& Astrophysics 365(1), L27-L35 (2001).

3 G. P. Garmire, M. W. Bautz, P. G. Ford, et al., "Advanced CCD imaging spectrometer (ACIS) instrument on the Chandra X-ray Observatory," in X-Ray and Gamma-Ray Telescopes and Instruments for Astronomy, 4851, 28-44, International Society for Optics and Photonics (2003).

4 B. LaMarr, M. W. Bautz, S. E. Kissel, et al., "Ground calibration of X-ray CCD detectors with charge injection for the X-ray imaging spectrometer on Astro-E2," in High-Energy Detectors in Astronomy, 5501, 385-391, International Society for Optics and Photonics (2004).

5 M. Bigas, E. Cabruja, J. Forest, et al., "Review of CMOS image sensors," Microelectronics journal 37(5), 433-451 (2006).

6 F. Scholze, H. Rabus, and G. Ulm, "Measurement of the mean electron-hole pair creation 
energy in crystalline silicon for photons in the 50-1500 eV spectral range," Applied physics letters 69(20), 2974-2976 (1996).

7 J. McChesney, R. Reininger, M. Ramanathan, et al., "The intermediate energy X-ray beamline at the APS," Nuclear Instruments and Methods in Physics Research Section A: Accelerators, Spectrometers, Detectors and Associated Equipment 746, 98-105 (2014).

8 N. Gehrels, "Confidence Limits for Small Numbers of Events in Astrophysical Data," The Astrophysical Journal 303, 336 (1986).

9 A. Owens, G. Fraser, and K. Mccarthy, "On the experimental determination of the Fano factor in Si at soft X-ray wavelengths," Nuclear Instruments and Methods in Physics Research Section A Accelerators Spectrometers Detectors and Associated Equipment 491, 437 (2002).

10 M. Bautz, S. Kissel, B. LaMarr, et al., "Improved x-ray CCD response at very low x-ray energies," in Space Telescopes and Instrumentation II: Ultraviolet to Gamma Ray, 6266, 62662P, International Society for Optics and Photonics (2006).

11 X. Chandra and R. Center, "Chandra Proposers' Observatory Guide," (2000).

12 M. S. Haro, F. A. Bessia, M. Pérez, et al., "Soft x-rays spectroscopy with a commercial cmos image sensor at room temperature," Radiation Physics and Chemistry 167, 108354 (2020).

13 P. Martin-Gonthier and P. Magnan, "RTS noise impact in CMOS image sensors readout circuit," in 2009 16th IEEE International Conference on Electronics, Circuits and Systems(ICECS 2009), 928-931, IEEE (2009).

14 H. Spieler, "Introduction to radiation-resistant semiconductor devices and circuits," in AIP Conference Proceedings, 390(1), 23-49, American Institute of Physics (1997). 